\title{
Vitellogenin and vitellogenin-like genes: not just for egg production
}

\author{
M. H. Richards ${ }^{1}$
}

Published online: 29 October 2019

(c) International Union for the Study of Social Insects (IUSSI) 2019

A lot of fascinating research on the complexities of social insects boils down to some very elementary questions: who lays eggs, how many do they lay, and what kinds of offspring develop from those eggs? Given the primary role of egg laying in social insect behaviour and evolution, it follows that the genetic mechanisms underpinning egg-laying behaviour must also be of central importance. While many genes are undoubtedly linked to egg-laying behaviour, one particular gene, vitellogenin, is of central importance. Vitellogenin literally means 'egg yolk producer'. The best known role of vitellogenin protein is as a precursor to vitellin protein, which is required for yolk formation, not only in social insects, but in all egg-laying animals. Although vitellogenin and its homologues in different animal lineages are sometimes referred to by different names, expression of the vitellogenin gene is clearly crucial for egg production. However, making eggs is not vitellogenin's only role. For instance, vitellogenin is involved in energy metabolism of glial cells in the honeybee brain (Münch et al. 2015) and is also expressed in drones (Colonello-Frattini et al. 2010), demonstrating that it has multiple functions, some of which are unrelated to egg production.

As a gene with multiple functions, the vitellogenin gene provides a textbook example of pleiotropy. Pleiotropic genes are interesting because selection on one of their functions necessarily influences their other functions. Gene duplication events may resolve this pleiotropy problem, because alternative copies of a gene can evolve separately and acquire functions different from those of the ancestral gene. For instance, paralogues of vitellogenin might specifically influence caste-associated behaviours other than egg laying. As it turns out, vitellogenin is evolutionarily prone to duplication. In addition to the conventional vitellogenin ( $v g$ ) gene, genome studies have uncovered three loci usually referred to as Vg-like-A, Vg-like-B, and Vg-like-C. Conventional $V g$,

M. H. Richards

mrichards@brocku.ca

1 Brock University, St. Catharines, Canada
$V g$-like- $A$, and $V g$-like- $B$ are found in all insects, implying very ancient gene duplications in arthropods, whereas $V g$ like-C is specific to Hymenoptera, implying more recent duplication. Ant genomes provide evidence for additional repeated and independent duplications of conventional $\mathrm{Vg}$ in different lineages, with some ants carrying as many as five copies of the conventional $\mathrm{Vg}$ gene (Kohlmeier et al. 2018).

In this issue, Claire Morandin and colleagues investigate the functional roles of conventional vitellogenin, $\mathrm{Vg}$-like$A, V g$-like- $B$, and $V g$-like- $C$ by comparing RNA expression levels among different social phenotypes in the ant, Formica fusca (Morandin et al. 2019). In general, RNA expression studies are based on the premise that a particular protein is produced when it is required, and that this requirement can be measured by quantifying the amount of RNA that would be available for protein translation. When individuals with alternative phenotypes, such as contrasting activity patterns or morphologies, exhibit differential RNA expression, we can associate gene expression patterns with the alternative phenotypes being investigated.

Morandin et al. (2019) compare gene expression patterns among ant social phenotypes that differ in egg-laying capacity, specifically queens vs. workers, nurse workers vs. foraging workers, and workers in queenless vs. queenright colonies. In each of these pairings, the first group lays more eggs than the second, so should express higher levels of conventional $V g$ RNA. In line with many previous studies, conventional $V g$ expression levels correlated with females' overall egg-laying capacity, being much higher in queens than in workers and higher in nurse workers than in foraging workers. However, queen removal did not have any effect, perhaps because worker egg-laying frequency increased only slightly. Overall, the correlation between conventional $V g$ expression and egg-laying frequency supports the general conception of this gene as primarily (but not exclusively) an egg yolk protein gene. In contrast, the expression patterns of the Vit-like genes differed from those of conventional $v g$. The biochemical roles of the Vit-like proteins are not known, so comparing their gene expression profiles to conventional $v g$ gene expression patterns might help to reveal 
their functions. In F. fusca, conventional $V g$ and $V g$-like- $A$ exhibited subtle differences in their expression patterns, the former being more highly expressed in queens than nurse workers, whereas the latter was similar in these two groups. In another recent study in Temnothorax longispinosus, knockdown of $V g$-like- $A$ expression changed how workers reacted to nestmates, high expression levels being associated with brood care and low expression with adult nestmate care (Kohlmeier et al. 2018). It would be interesting to compare the expression patterns of these two genes in social insects in which females are simultaneously involved in egg laying and brood care.

In stark contrast to the Vg-like-A results, Morandin et al. (2019) found that in F. fusca, both Vg-like-B and $C$ were elevated in foraging workers compared to nurses and queens. These expression patterns support the view that Vit-like $B$ and $C$ have diverged more strongly from the original functions of the $V g$ family than $V g$-like-A. A possible role for Vit-like-C in regulating social behaviour is particularly intriguing. In the current study, $\mathrm{Vg}$-like-C was expressed more strongly in foragers than nurses or queens. This pattern reflects that seen in six additional Formica species (Morandin et al. 2014), and in the bumblebee Bombus terrestris, where it is more highly expressed in nonreproductive workers than in queens (Harrison et al. 2015). Vit-like-C is found only in Hymenoptera, and its predicted protein structure is so truncated that it cannot function as a lipid-binding molecule (Morandin et al. 2014), which in itself is evidence that this gene has acquired completely novel functions unrelated to lipid transport or egg production. It would seem to be a promising candidate for further studies in both solitary and social hymenopterans, perhaps modelled after the comprehensive comparisons described by Morandin et al. 2019.

\section{References}

Colonello-Frattini NA, Guidugli-Lazzarini KR, Simões ZLP, Hartfelder K (2010) Mars is close to Venus - female reproductive proteins are expressed in the fat body and reproductive tract of honey bee (Apis mellifera L.) drones. J Insect Physiol 56:1638-1644

Harrison MC, Hammond RL, Mallon EB (2015) Reproductive workers show queenlike gene expression in an intermediately eusocial insect, the buff-tailed bumble bee Bombus terrestris. Mol Ecol 24:3043-3063

Kohlmeier P, Feldmeyer B, Foitzik S (2018) Vitellogenin-like Aassociated shifts in social cue responsiveness regulate behavioral task specialization in an ant. PLoS Biol 16(6):e2005747

Morandin C, Havukainen H, Kulmuni J, Dhaygude K, Trontti K, Helantera $\mathrm{H}$ (2014) Not only for egg yolk-functional and evolutionary insights from expression, selection, and structural analyses of Formica Ant vitellogenins. Mol Biol Evol 31:2181-2193

Morandin C, Hietala A, Helanterä H (2019) Vitellogenin and vitellogenin-like gene expression patterns in relation to caste and task in the ant Formica fusca. Insect Soc. https://doi.org/10.1007/s0004 0-019-00725-9

Münch D, Ihle KE, Salmela H, Amdam GV (2015) Vitellogenin in the honey bee brain: atypical localization of a reproductive protein that promotes longevity. Exp Gerontol 71:103-108 